\title{
Reduced urinary levels of angiotensin-converting enzyme 2 activity predict acute kidney injury in critically ill patients
}

Laurent Bitker, Sheila K Patel, Intissar Bittar, Glenn M Eastwood, Rinaldo Bellomo and Louise M Burrell www.doi.org/10.51893/2020.4.0a7

Published online first 7 December 2020

Acute kidney injury (AKI) is common in patients admitted to the intensive care unit (ICU), yet our understanding of its pathophysiology is poor. ${ }^{1} \mathrm{AKI}$ increases the risk of death of critically ill patients, proportionally to its severity, and has both short term and long-lasting consequences on patients' quality of life..$^{2-4}$ Suspected mechanisms leading to an acute fall in renal function include a combination of intrarenal macrovascular and microvascular mismatch associated with inflammation and metabolic stress. ${ }^{1,5-7}$

The non-classical renin-angiotensin system (RAS) is a recently identified endocrine cascade, with regulating effects on renal function and haemodynamics.8,9 The pivotal enzyme of the non-classical RAS is angiotensinconverting enzyme 2 (ACE2). ${ }^{10-12}$ The major role of this transmembrane monocarboxypeptidase is to convert angiotensin II into angiotensin. ${ }^{1-7}$ ACE2 is highly expressed in the heart and kidneys, and is also present in the lungs and brain. 9,13,14 ACE2 is also the receptor for cell entry of severe acute respiratory syndrome coronavirus 2 (SARS-CoV-2). ${ }^{15}$ In the kidney, ACE2 induces vasodilation and nitric oxide release, and increases renal blood flow, glomerular filtration, natriuresis and diuresis, while downregulating inflammatory and profibrogenic pathways. ${ }^{16-20}$ Thus, increased expression of ACE2 in the kidney could be associated with decreased risk of $\mathrm{AKI}$.

Because ACE2 is expressed mainly in proximal tubular epithelial cells, and can be cleaved from the cell surface into the urine, quantification of ACE2 activity levels in human urine may contribute to our understanding of the role played by the intrarenal non-classical RAS in critically ill patients at risk of AKI. ${ }^{21}$ Consequently, we evaluated the association of urinary ACE2 (UACE2) activity with AKI, and performance of UACE2 activity in predicting AKI, in a general ICU population. We hypothesised that increased UACE2 activity would be associated with a lower risk of AKI in the acute care setting.

\section{Methods}

This single-centre prospective, observational, exploratory study was approved by the Austin Health Human Research Ethics Committee (Melbourne, Australia, approval number LNR/18/Austin/151 for patients and LNRSSA/18/

\section{ABSTRACT}

Objective: Angiotensin-converting enzyme 2 activity reflects non-classical renin-angiotensin system upregulation. We assessed the association of urinary angiotensin-converting enzyme 2 (UACE2) activity with acute kidney injury (AKI).

Design, setting and participants: A prospective observational study in which we measured UACE2 activity in 105 critically ill patients at risk of AKI. We report AKI stage 2 or 3 at 12 hours of urine collection $\left(A K I_{12 h}\right)$ and AKI stage 2 or 3 at any time during intensive care unit stay in patients free from any stage of AKI at inclusion $\left(\mathrm{AKI}_{\mathrm{ICU}}\right)$. AKI prediction was assessed using area under the receiver-operating characteristics curve (AUROC) and net reclassification indices (NRIs).

Main outcome measure: AKI stage 2 or 3 at 12 hours of urine collection.

Results: Within 12 hours of inclusion, 32 of 105 patients $(30 \%)$ had developed $\mathrm{AKI}_{12 \mathrm{~h}}$. Corrected UACE2 activity was significantly higher in patients without $\mathrm{AKI}_{12 \mathrm{~h}}$ compared with those with $\mathrm{AKI}_{12 \mathrm{~h}}$ (median [interquartile range], 13 [624] 7 [4-10] $\mathrm{pmol} / \mathrm{min} / \mathrm{mL}$ per $\mathrm{mmol} / \mathrm{L}$ of urine creatinine; $P<0.01)$. A 10-unit increase in UACE2 was associated with a $28 \%$ decrease in $\mathrm{AKI}_{12 \mathrm{~h}}$ risk (odds ratio [95\% Cl], 0.72 [0.46-0.97]). During intensive care unit admission, 39 of 76 patients ( $51 \%$ ) developed AKI ICU. UACE2 had an AUROC for the prediction of $\mathrm{AKI}_{12 \mathrm{~h}}$ of $0.68(95 \% \mathrm{Cl}, 0.57-0.79)$, and correctly reclassified $28 \%$ of patients (positive NRI) to $\mathrm{AKI}_{12 \mathrm{~h}}$. Patients with UACE2 $>8.7 \mathrm{pmol} / \mathrm{min} / \mathrm{mL}$ per $\mathrm{mmol} / \mathrm{L}$ of urine creatinine had a significantly lower risk of $A K I_{\mathrm{ICU}}$ on log-rank analysis (52\% v 84\%; $P<0.01)$.

Conclusions: Higher UACE2 activity was associated with a decreased risk of AKI stage 2 or 3 . Our findings support future evaluations of the role of the non-classical reninangiotensin system during AKI.

Crit Care Resusc 2020; 22 (4): 344-354

Austin/315 for healthy volunteers), who waived the need for informed consent for the collection of urine in the critically ill population. The study protocol and report met the STROBE criteria for observational studies and the STARD 


\section{ORIGINAL ARTICLES}

recommendations for reporting diagnostic accuracy studies (Online Appendix, methods). 22,23

\section{Study cohort}

We prospectively enrolled a convenience sample of all consecutive adult patients admitted to the Department of Intensive Care at Austin Hospital, within 48 hours of their admission, and presenting one of the following inclusion criteria in the preceding 6 hours: cardiovascular sepsisrelated organ failure assessment (SOFA) score $\geq 1$, respiratory SOFA $\geq 2$, increase in serum creatinine level $>8 \mu \mathrm{mol} / \mathrm{L}$ between two creatinine measurements performed during the 6-hour period preceding enrolment, or a urine output $<0.5 \mathrm{~mL} / \mathrm{kg} / \mathrm{h}$ over 4 hours during the same 6 -hour block. ${ }^{24}$

The first two inclusion criteria were those used in the Sapphire study, a landmark study on AKI risk prediction. ${ }^{25}$ The other two were selected to target patients at risk of $\mathrm{AKI}$; they were derived from the AKI stage 1 definition of the Kidney Disease: Improving Global Outcomes (KDIGO) recommendations, to reflect a potentially small yet significant change in renal function over a limited period, ${ }^{26}$ and have been recently validated. ${ }^{27}$

We excluded patients with anuria, known stage 2 or $3 \mathrm{AKI}$ at the time of enrolment (including those on renal replacement therapy [RRT]), stage 4 or 5 chronic kidney disease (including renal transplant recipients and those on maintenance dialysis), history of urinary tract surgery, expected length of stay $<48$ hours, and patients undergoing end-of-life care.

\section{Urine collection and handling}

Immediately after inclusion, we collected a $10 \mathrm{~mL}$ spot urine sample from the indwelling vesical catheter. Urine collection was performed on weekdays, between 8:00 am and $12: 00 \mathrm{pm}$. Samples were then centrifuged at $1500 \times \mathrm{g}$ for 10 minutes, and $0.5 \mathrm{~mL}$ of urine supernatant was aliquoted and stored at $-80^{\circ} \mathrm{C}$. No adjuvant was added to the urine sample before storage.

\section{Quantification of UACE2 activity}

Urine ACE2 activity was measured using a sensitive quenched fluorescent substrate-based assay, ${ }^{21}$ with modifications. Urine was incubated in triplicate with an ACE2-specific quenched fluorescent substrate (QFS): (7-methoxycoumarin-4-yl)-acetyl-Ala-Pro-Lys(2,4dinitrophenyl) (Auspep, Melbourne, Vic, Australia), with or without an ACE2 inhibitor mix. Assays were performed with $50 \mu \mathrm{M}$ of QFS, in a final volume of $200 \mu \mathrm{L}$ per well, with ACE2 assay buffer (100 mM Tris, $1 \mathrm{M} \mathrm{NaCl}, \mathrm{pH} 6.5)$. Reactions were performed at $37^{\circ} \mathrm{C}$ for 200 minutes with continuous monitoring of liberated fluorescence using a FLUOstar OPTIMA plate reader (BMG Labtech, Offenburg,
Germany). Cleavage of the QFS was attributed to ACE2 by the use of a specific inhibitor mix containing the ACE2 inhibitor MLN-4760 (MSD, Sydney, NSW, Australia) at a final concentration of $1 \mu \mathrm{M}, 10 \mu \mathrm{M}$ Z-pro (Bachem, Bubendorf, Switzerland) and $10 \mathrm{mM}$ EDTA (Sigma-Aldrich, Sydney, NSW, Australia). A protease inhibitor cocktail was also added comprising $0.0412 \mathrm{M}$ of $\mathrm{N}$-ethylmaleimide, $840 \mathrm{U} / \mathrm{mL}$ of Aprotinin, $2.1 \mathrm{UM}$ of Leupeptin and $0.3 \mu \mathrm{M}$ of Pepstatin A. The rate of substrate cleavage was determined by comparison with a standard curve of the free fluorophore 4-aminomethoxycoumarin (Sigma-Aldrich) and expressed as pmol of substrate cleaved $/ \mathrm{min} / \mathrm{mL}$ of urine. The intra-assay and inter-assay coefficients of variation were $5.7 \%$ and $9.4 \%$, respectively. The investigators measuring UACE2 activity were blinded to the clinical primary outcome adjudication.

To adjust UACE2 activity to varying urine flow rate and density, we normalised UACE2 levels to urine creatinine levels $(\mathrm{UCr})$; hence, all presented $\mathrm{UACE} 2$ data were $\mathrm{UCr}$-corrected unless otherwise specified. ${ }^{28,29}$ To do so, we measured $\mathrm{uCr}$ levels (in $\mathrm{mmol} / \mathrm{L}$ ) on the same urine spot sample (cobas 8000 analyser, Roche Diagnostics, Indianapolis, Ind, USA). Corrected UACE2 activity was hence expressed in pmol/min/ $\mathrm{mL}$ per $\mathrm{mmol} / \mathrm{L}$ of $\mathrm{UCr}$.

\section{Primary and secondary outcomes}

For the present study, we defined the primary outcome as the occurrence of AKI stage 2 or 3 (following the KDIGO guidelines staging system) at 12 hours of urine collection. ${ }^{26}$ To do so, we recorded 12-hour cumulative urine output after urine collection and used the serum creatinine value measured closest to the 12-hour time point (using a 2-hour bilateral time window). Adjudication of the primary outcome was performed before UACE2 results were made available to investigators. If adjudication of the primary outcome was impossible, the data were excluded from the analysis.

Secondary renal outcomes were any AKI stage at 12 hours (KDIGO stage 1 to 3 ), RRT during the index ICU admission, AKI stage 2 or 3 on the day following urine collection (at 24 hours), and highest AKI stage during the index ICU admission up to 7 days after inclusion or death. Elapsed time between urine collection and the highest AKI stage was also recorded.

\section{Assessment of acute renal function}

Urine output was normalised to patient weight. Serum creatinine concentration was measured with our point-ofcare blood gas analyser (Radiometer ABL800, Radiometer Medical ApS, Copenhagen, Denmark). ${ }^{30}$ If this measurement was missing, we used that measured by the Austin Health Department of Pathology.

Premorbid serum creatinine level was assessed using all available data present in the electronic medical record, and 


\section{ORIGINAL ARTICLES}

corresponded to the lowest serum creatinine level measured between 365 days and 7 days before ICU admission, and closest to the latter. If this information was unavailable, we retrospectively estimated it by reporting the lowest stable serum creatinine level recorded during the index admission. Premorbid estimated glomerular filtration rate was systematically re-estimated using the modified diet in renal disease formula, to avoid bias relating to differing estimation methods used in result reports. ${ }^{31}$

\section{Other patient characteristics}

We recorded patient demographics, comorbidities, category and origin of ICU admission, severity of illness as assessed by APACHE (Acute Physiology and Chronic Health Evaluation) III and SOFA scores, characteristics of organ failure and support, premorbid treatment with ACE inhibitors or angiotensin receptor blockers, and treatment with diuretics before urine collection. ${ }^{24,32}$

\section{Healthy volunteer study}

To compare UACE2 activity to that of a non-critically ill population, we collected $10 \mathrm{~mL}$ of urine from 10 healthy volunteers between 8:00 am and 12:00 pm, and analysed these samples according to the protocol used for patient samples.

\section{Statistical analysis}

We analysed data using the R software (version 3.3.1, R Foundation for Statistical Computing, Vienna, Austria), with the survival, pROC and nricens packages. ${ }^{33-35}$ A $P$ value below 0.05 was considered statistically significant. Unless stated otherwise, continuous variables are expressed as median with interquartile range (IQR) and compared between groups using the Wilcoxon-MannWhitney test, and categorical variables are expressed as count with percentage and compared between groups using the Fisher exact test. No imputation for missing data was performed. A convenience sample size of at least 100 UACE2 measurements was chosen, as sample size calculation was unfeasible due to the absence of previous reference UACE2 data in this population.

We assessed the association of UACE2 activity with AKI risk, using generalised linear models. We then performed a predetermined sensitivity analysis, after exclusion of oliguric (non-anuric) patients at time of urine collection (defined as a urine output of $<0.5 \mathrm{~mL}$ $\mathrm{kg} / \mathrm{h}$ over 6 hours). Adjustment for AKI risk was performed using the following predefined variables: age, premorbid serum creatinine levels and APACHE III score (the reference model). Model variables were selected following the Sapphire study, which studied the predictive performance of AKI biomarkers, using an AKI reference model. ${ }^{25}$ The calibration of the reference model was assessed using the Pearson $\chi^{2}$ test. Time to AKI stage 2 or 3 during ICU stay was analysed using the logrank test, after dichotomising the study population based on the observed median UACE2 value.

To assess the biomarker's performance to predict AKI, we pre-specified the computation of the area under the receiver-operating characteristics curve (AUROC) of UACE2 activity corrected for $\mathrm{UCr}$ for the detection of the primary outcome. A similar method was applied to the analysis of secondary outcomes and to the assessment of the adjusted AKI risk models. We completed this analysis by calculating the net reclassification index (NRI) of UACE2, with the aim of reclassifying patients into three risk categories of the primary outcome (low $<5 \%$, medium $5 \%$ to $<30 \%$, high $\geq 30 \%$ ), using the same variables as described for the reference risk model. ${ }^{36}$ For exploratory purposes, we also determined the optimal cut-off value of UACE2, using the Youden criterion. From this, we computed the sensitivity, specificity and other relevant parameters of diagnostic accuracy.

\section{Results}

Between February 2018 and October 2018, we enrolled 105 critically ill patients (Figure 1). Inclusion (ie, time of urine
Figure 1. Study flow chart

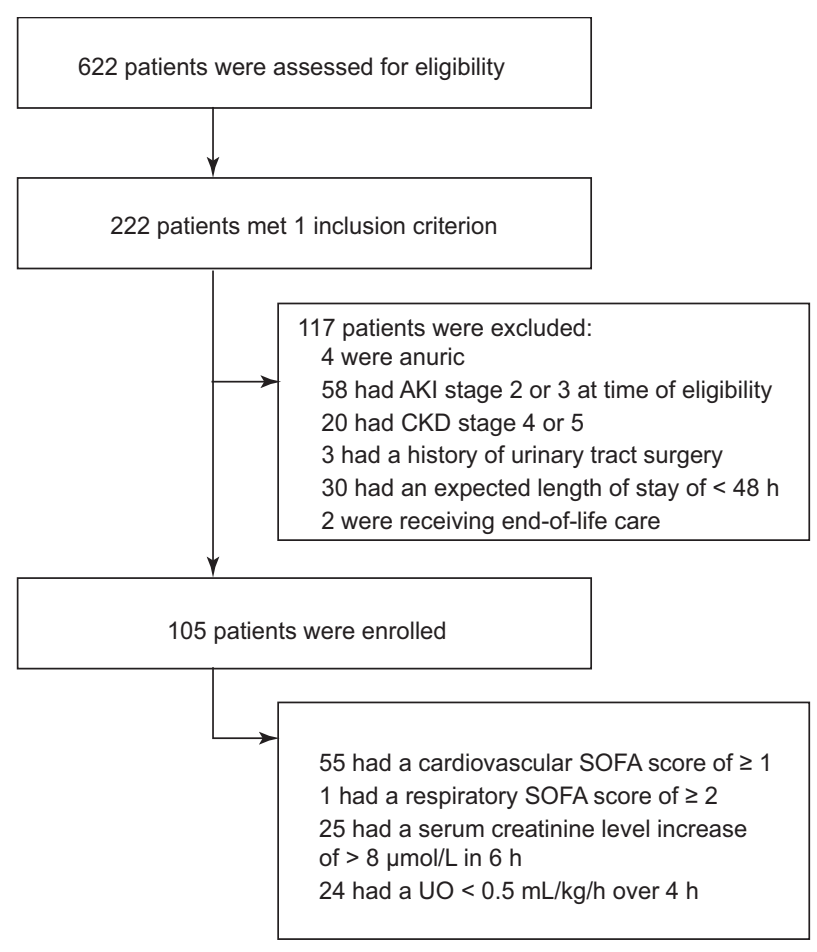

$A K I=$ acute kidney injury. $C K D=$ chronic kidney disease. SOFA = Sepsis-related Organ Failure Assessment. 


\begin{tabular}{|c|c|c|c|c|}
\hline & $\begin{array}{l}\text { Whole cohort } \\
\qquad(N=105)\end{array}$ & $\begin{array}{l}\text { AKI- at } 12 \mathrm{~h} \\
(n=73)\end{array}$ & $\begin{array}{l}\mathrm{AKI}+\text { at } 12 \mathrm{~h} \\
(n=32)\end{array}$ & $P$ \\
\hline \multicolumn{5}{|l|}{ Demographics } \\
\hline Sex (male) & $56(53 \%)$ & $37(51 \%)$ & $19(59 \%)$ & 0.52 \\
\hline Age (years) & $64(51-74)$ & $64(52-74)$ & $64(51-73)$ & 0.85 \\
\hline \multicolumn{5}{|l|}{ Comorbidities } \\
\hline Hypertension & $53(50 \%)$ & $35(48 \%)$ & $18(56 \%)$ & 0.53 \\
\hline Diabetes & $22(21 \%)$ & $14(19 \%)$ & $8(25 \%)$ & 0.60 \\
\hline Peripheral vascular disease & $12(11 \%)$ & $7(10 \%)$ & $5(16 \%)$ & 0.51 \\
\hline Ischaemic heart disease & $25(24 \%)$ & $20(27 \%)$ & $5(16 \%)$ & 0.22 \\
\hline Chronic heart disease & $10(10 \%)$ & $8(11 \%)$ & $2(6 \%)$ & 0.72 \\
\hline Cerebrovascular disease & $8(8 \%)$ & $6(8 \%)$ & $2(6 \%)$ & $>0.99$ \\
\hline Chronic obstructive pulmonary disease & $16(15 \%)$ & $12(16 \%)$ & $4(12 \%)$ & 0.77 \\
\hline Chronic liver disease & $15(14 \%)$ & $9(12 \%)$ & $6(19 \%)$ & 0.38 \\
\hline Use of ACE inhibitors & $15(14 \%)$ & $12(16 \%)$ & $3(9 \%)$ & 0.55 \\
\hline \multicolumn{5}{|l|}{ Premorbid renal function } \\
\hline Serum creatinine $(\mu \mathrm{mol} / \mathrm{L})$ & $76(57-89)$ & $69(54-86)$ & $86(73-100)$ & $<0.01$ \\
\hline $\mathrm{eGFR}\left(\mathrm{mL} / \mathrm{min} / 1.73 \mathrm{~m}^{2}\right)$ & $90(64-90)$ & $90(74-90)$ & $80(56-90)$ & $<0.01$ \\
\hline \multicolumn{5}{|l|}{ ICU admission category } \\
\hline Elective surgery & $27(26 \%)$ & $18(25 \%)$ & $9(28 \%)$ & 0.01 \\
\hline Emergent surgery & $31(30 \%)$ & $16(22 \%)$ & $15(47 \%)$ & - \\
\hline Medical & $47(45 \%)$ & $39(53 \%)$ & $8(25 \%)$ & - \\
\hline \multicolumn{5}{|l|}{ ICU admission subgroup } \\
\hline Cardiac surgery & $16(15 \%)$ & $11(15 \%)$ & $5(16 \%)$ & 0.13 \\
\hline Neurologic admission & $14(13 \%)$ & $9(12 \%)$ & $5(16 \%)$ & 0.13 \\
\hline \multicolumn{5}{|l|}{ Severity of disease } \\
\hline APACHE III score & $47(40-64)$ & $47(38-64)$ & $51(42-61)$ & 0.39 \\
\hline SOFA score & $6(4-8)$ & $6(5-8)$ & $6(4-9)$ & 0.79 \\
\hline Vasopressor support & $69(66 \%)$ & $48(66 \%)$ & $21(66 \%)$ & $>0.99$ \\
\hline Mechanical ventilation & $73(70 \%)$ & $52(71 \%)$ & $21(66 \%)$ & 0.65 \\
\hline Sepsis & $32(30 \%)$ & $25(34 \%)$ & $7(22 \%)$ & 0.25 \\
\hline \multicolumn{5}{|l|}{ Blood results at inclusion ${ }^{+}$} \\
\hline Highest white cell count $\left(\times 10^{9} / \mathrm{L}\right)$ & $13.1(10.2-16.9)$ & $13.3(10.2-15.7)$ & $13(10.5-17.6)$ & 0.65 \\
\hline Highest lactate (mmol/L) & $2.6(1.6-4.4)$ & $2.6(1.6-4.2)$ & $2.5(1.8-4.5)$ & 0.86 \\
\hline \multicolumn{5}{|l|}{$\begin{array}{l}\text { Renal function and related characteristics at } \\
\text { inclusion }\end{array}$} \\
\hline Serum creatinine $(\mu \mathrm{mol} / \mathrm{L})$ & $87(71-122)$ & $80(67-110)$ & $102(81-158)$ & $<0.01$ \\
\hline Estimated baseline creatinine & $45(43 \%)$ & $29(40 \%)$ & $16(50 \%)$ & 0.39 \\
\hline Urine output $(\mathrm{mL} / \mathrm{kg} / \mathrm{h})^{\ddagger}$ & $0.7(0.4-1.0)$ & $0.8(0.5-1.1)$ & $0.4(0.3-0.7)$ & $<0.01$ \\
\hline Fluid balance $(\mathrm{mL} / \mathrm{kg} / \mathrm{h})^{\ddagger}$ & $0(-0.8$ to 0.6$)$ & $-0.2(-0.8$ to 0.6$)$ & $0.1(-0.6$ to 0.6$)$ & 0.52 \\
\hline Diuretic use $\mathrm{e}^{\S}$ & $13(12 \%)$ & $12(16 \%)$ & $1(3 \%)$ & 0.10 \\
\hline Prior use of ACE inhibitors" & $15(14 \%)$ & $12(16 \%)$ & $3(9 \%)$ & 0.54 \\
\hline Prior use of ARBs" & $21(20 \%)$ & $12(16 \%)$ & $9(28 \%)$ & 0.19 \\
\hline
\end{tabular}




\section{ORIGINAL ARTICLES}

Table 1. Patient characteristics based on absence or presence of AKI stage 2 or 3 at 12 hours* (continued)

\begin{tabular}{|c|c|c|c|c|}
\hline & $\begin{array}{l}\text { Whole cohort } \\
\qquad(N=105)\end{array}$ & $\begin{array}{c}\text { AKI- at } 12 \mathrm{~h} \\
(n=73)\end{array}$ & $\begin{array}{c}\mathrm{AKI}+\text { at } 12 \mathrm{~h} \\
(n=32)\end{array}$ & $P$ \\
\hline \multicolumn{5}{|l|}{ ICU-related outcomes } \\
\hline Length of stay (day) & $3(2-6)$ & $3(2-6)$ & $4(2-6)$ & 0.56 \\
\hline Duration of mechanical ventilation (h) & $26(10-69)$ & $25(11-64)$ & $29(6-96.5)$ & 0.99 \\
\hline Duration of vasopressor support (h) & $18(2-39)$ & $20(8-41)$ & $12.5(0-35)$ & 0.14 \\
\hline ICU mortality & $11(10 \%)$ & $9(12 \%)$ & $2(6 \%)$ & 0.50 \\
\hline
\end{tabular}

$\mathrm{ACE}=$ angiotensin-converting enzyme. $\mathrm{AKI}=$ acute kidney injury. $\mathrm{AKI}-=\mathrm{AKI}$ stage 0 or 1 at $12 \mathrm{~h}$ of urine collection. $\mathrm{AKI}+=\mathrm{AKI}$ stage 2 or 3 at $12 \mathrm{~h}$ of urine collection. APACHE = Acute Physiology and Chronic Health Evaluation. ARB = angiotensin receptor blocker. eGFR = estimated glomerular filtration rate. ICU = intensive care unit. SOFA = sepsis-related organ failure assessment. * Data are median (interquartile range) or count (percentage). + Measured in the $24 \mathrm{~h}$ preceding inclusion. ₹ Recorded over the $6 \mathrm{~h}$ preceding inclusion. $\S$ Administered within the $6 \mathrm{~h}$ preceding inclusion. 9 Defined as the prescription of ACE inhibitors or ARBs in the most recent history.

collection) occurred at a median of 14 (IQR, 10-17] hours of ICU admission. The patients' characteristics are summarised in Table 1. Co-existence of inclusion criteria is shown in the Online Appendix (table S1). Adjudication of the primary outcome was possible for all 105 included patients.

\section{Corrected UACE2 activity in ICU patients and healthy volunteers}

The median corrected UACE2 activity for the study cohort was $8.7(I Q R$, 5.4-19.1) $\mathrm{pmol} / \mathrm{min} / \mathrm{mL}$ per $\mathrm{mmol} / \mathrm{L}$ of $\mathrm{uCr}$ $(N=105)$, which was similar to that for the healthy volunteers (Online Appendix, table S2). No difference in UACE2 activity was observed based on study inclusion criteria (Online Appendix, figure S1). UACE2 activity was similar in patients on ACE inhibitors (7.8 [6.1-20.3] pmol/min/mL per mmol/L of $\mathrm{uCr}$ ) to those treated with angiotensin receptor blockers (8.0 [6.1-14.5] pmol/min/mL per $\mathrm{mmol} / \mathrm{L}$ of $\mathrm{UCr}$ ) and those not treated with either of these classes of RAS inhibitors (9.4 [5.4-19.7] pmol $/ \mathrm{min} / \mathrm{mL}$ per $\mathrm{mmol} / \mathrm{L}$ of $\mathrm{uCr})(P=0.98$, Kruskall-Wallis test across the three groups).

\section{AKI incidence and severity}

Within 12 hours of inclusion, 32 of 105 patients (30\%) had developed AKI stage 2 or 3 . Their premorbid renal function and renal function at time of inclusion significantly differed from patients who did not develop the primary outcome (Table 1). The characteristics of the primary and secondary outcomes are shown in Table 2. Oliguria was the main AKI defining criterion; seven of 25 patients with oliguria-defined AKI showed a subsequent increase in serum creatinine level greater than 1.5 times more than the baseline value during their ICU stay.

\section{Corrected UACE2 activity in patients with AKI}

Corrected UACE2 activity in patients who developed the primary outcome was significantly lower compared with that for those who did not develop the primary outcome, and comparable to measurements in healthy volunteers (Figure 2, panel A). For patients without AKI stage 2 or 3 at $12 \mathrm{~h}$ versus those with AKI stage 2 or 3 at $12 \mathrm{~h}$, the median [interquartile range] corrected UACE2 activity values were $13[6-24]$ and 7 [4-10] pmol/min/mL per mmol/L of urine creatinine, respectively $(P<0.01)$. UACE2 levels based on AKI status for each inclusion criterion are shown in the Online Appendix (table S3).

Association of corrected UACE2 activity with risk of severe AKI

On unadjusted analysis, higher UACE2 activity was associated with a $28 \%$ decrease in risk of AKI stage 2 or 3 at 12 hours (OR, $0.72[95 \% \mathrm{Cl}, 0.46-0.97]$ for each $10 \mathrm{pmol} / \mathrm{min} / \mathrm{mL}$ per $\mathrm{mmol} / \mathrm{L}$ of $\mathrm{uCr}$ increase in concentration) (Figure 3). This persisted after adjustment for the predefined covariates (Table 3). The significant association between UACE2 levels and the primary outcome remained in patients without oliguria at inclusion (Online Appendix, figure S2).

In the subgroup of patients without any degree of AKI before inclusion, 39 of $76(51 \%)$ developed AKI stage 2 or 3 at any time during ICU admission, at a median time of 13 (IQR, 10-52) hours after inclusion. Those with UACE2 activity $>8.7 \mathrm{pmol} /$ $\mathrm{min} / \mathrm{mL}$ per $\mathrm{mmol} / \mathrm{L}$ of $\mathrm{UCr}$ experienced a lower time-weighted risk of AKI stage 2 or 3 during ICU admission (Figure 4).

\section{Performance of UACE2 as a predictor of risk of AKI}

In isolation, UACE2 showed a poor, yet significant, discriminative ability to predict AKI stage 2 or 3 at 12 hours, 
Table 2. Analysis of primary outcome and secondary renal outcomes for AKI- and AKI+ patients*

\begin{tabular}{|c|c|c|c|}
\hline & $\begin{array}{c}\text { AKI- } \\
(n=73)\end{array}$ & $\begin{array}{c}\text { AKI+ } \\
(n=32)\end{array}$ & $P$ \\
\hline \multicolumn{4}{|c|}{ Primary outcome (AKI stage 2 or 3 at $12 \mathrm{~h}$ ) } \\
\hline AKI stage ${ }^{\dagger}$ & & & - \\
\hline Stage 1 & $18(25 \%)$ & 0 & \\
\hline Stage 2 & 0 & $30(94 \%)$ & \\
\hline Stage 3 & 0 & $2(6 \%)$ & \\
\hline \multicolumn{4}{|l|}{ Renal function indices at $12 \mathrm{~h}$} \\
\hline Serum creatinine $(\mu \mathrm{mol} / \mathrm{L})$ & $80.0(64.0-102.2)$ & $109.0(87.0-187.5)$ & $<0.01$ \\
\hline Urine output (mL/kg/h) & $0.9(0.7-1.4)$ & $0.4(0.3-0.5)$ & $<0.01$ \\
\hline AKI defining criterion ${ }^{\dagger}$ & & & - \\
\hline Serum creatinine alone & - & $2(6 \%)$ & \\
\hline Urine output alone & - & $25(78 \%)$ & \\
\hline Combination of both & - & $5(16 \%)$ & \\
\hline \multicolumn{4}{|l|}{ Secondary renal outcomes } \\
\hline Renal replacement therapy & $3(4 \%)$ & $4(12 \%)$ & 0.20 \\
\hline AKI stage 2 or 3 at $24 \mathrm{~h}$ & $4(5 \%)$ & $17(53 \%)$ & $<0.01$ \\
\hline AKI stage 2 or 3 at any time & $7(10 \%)$ & $32(100 \%)$ & $<0.01$ \\
\hline Time to highest AKI stage (h) & $2.2(-4.9$ to 59.2$)$ & $12.1(11.0-13.1)$ & 0.11 \\
\hline
\end{tabular}

$\mathrm{AKI}=$ acute kidney injury. AKI- $=\mathrm{AKI}$ stage 0 or 1 at $12 \mathrm{~h}$ of urine collection. AKI+ $=$ AKI stage 2 or 3 at $12 \mathrm{~h}$ of urine collection. $\mathrm{KDIGO}=\mathrm{Kidney}$ Disease: Improving Global Outcomes. * Data are median (interquartile range) or count (percentage). † According to KDIGO guidelines.

Table 3. Adjusted predictive performance of UACE2 activity in critically ill patients for the primary outcome and other renal outcomes, for an increase in UACE2 level of $10 \mathrm{pmol} / \mathrm{min} / \mathrm{mL} \mathrm{per} \mathrm{mmol} / \mathrm{L}$ of $\mathrm{uCr}$

\begin{tabular}{|c|c|c|c|c|c|}
\hline Outcome & $\begin{array}{c}\text { Patients/ } \\
\text { total }\end{array}$ & $\begin{array}{c}\text { Adjusted OR } \\
(95 \% \mathrm{Cl}) *\end{array}$ & $\begin{array}{c}\text { AUROC } \\
(95 \% \mathrm{CI})\end{array}$ & $\mathrm{NRI}{ }^{+}$ & NRI- ${ }^{+}$ \\
\hline \multicolumn{6}{|l|}{ Primary outcome } \\
\hline AKI stage 2 or 3 at $12 \mathrm{~h}$ & $32 / 105$ & $0.65(0.40-0.90)$ & $0.75(0.65-0.84)$ & 0.28 & 0.03 \\
\hline \multicolumn{6}{|l|}{ Other renal outcomes } \\
\hline $\mathrm{AKI}$ (any stage) at $12 \mathrm{~h}$ & $50 / 105$ & $0.84(0.69-1.00)$ & $0.80(0.71-0.88)$ & - & - \\
\hline AKI stage 2 or 3 at $24 \mathrm{~h}$ & 25/105 & $1.05(0.89-1.22)$ & $0.70(0.57-0.84)$ & - & - \\
\hline AKI stage 2 or 3 at any time & $39 / 105$ & $0.93(0.77-1.08)$ & $0.74(0.64-0.84)$ & - & - \\
\hline
\end{tabular}

$\mathrm{AKI}=$ acute kidney injury. $\mathrm{APACHE}=$ Acute Physiology and Chronic Health Evaluation. AUROC = area under the receiver operator characteristics curve $\mathrm{NRI}=$ net reclassification index. $\mathrm{OR}=$ odds ratio. $\mathrm{UACE} 2=$ urinary angiotensin-converting enzyme type $2 . \mathrm{uCr}=$ urinary creatinine. ${ }^{*}$ Adjusted for age, premorbid serum creatinine levels and APACHE III score, except for the OR for AKI stage 2 or 3 at 24 h, which was adjusted for premorbid serum creatinine levels and APACHE III score only, owing to a limited number of events. Renal replacement therapy is not presented due to the limited number of cases $(<10)$. There were no missing data for the following variables that were included in the model: severe AKI at $12 \mathrm{~h}$, uACE2 measurements, age, premorbid serum creatinine levels, and APACHE III score. The reference model has an AUROC of $0.71(95 \% \mathrm{Cl}, 0.61-0.80)$, with no significant difference compared with the same model to which was added UACE2 activity $(P=0.36)$. Calibration was acceptable $\left(P=0.51\right.$, Pearson $\chi^{2}$ test). No significant interaction between independent terms of the reference model was identified. † To reclassify patients into three risk categories (low $<5 \%$, medium $5 \%$ to $<30 \%$, and high $\geq 30 \%)$, no NRI was computed if no statistical difference existed between the reference model and the model including UACE2 levels.

with an AUROC of $0.68(95 \% \mathrm{Cl}, 0.57-0.79)$. Discriminative performance became fair after exclusion of patients who were oliguric at time of urine collection (AUROC, 0.71 $[95 \% \mathrm{Cl}, 0.57-0.86])$.
The multivariate model (using UACE2 activity, age, premorbid serum creatinine level and APACHE III score) had an AUROC of $0.75(95 \% \mathrm{Cl}, 0.65-0.84)$ to predict AKI stage 2 or 3 at 12 hours (fair discriminative ability). UACE2 
Figure 2. Levels of uACE2 in critically ill patients with $A K I^{*}$

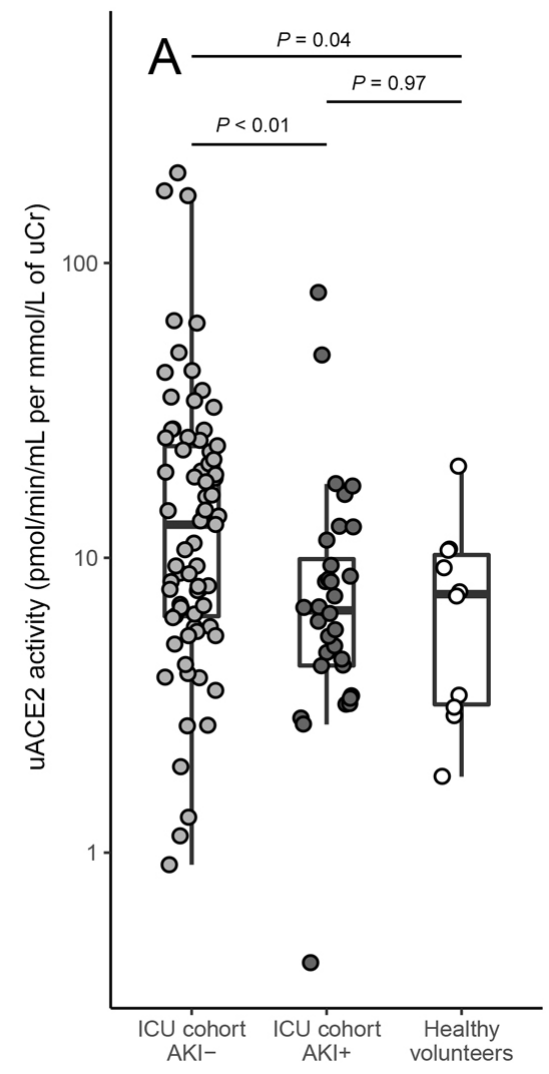

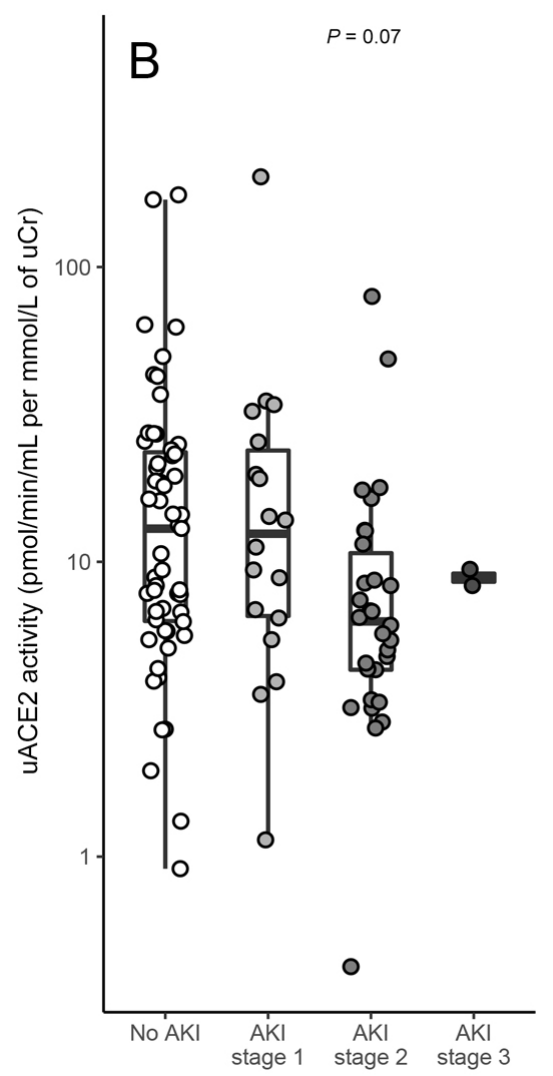

$\mathrm{AKI}=$ acute kidney injury. $\mathrm{AKI}-=\mathrm{AKI}$ stage 0 or 1 at $12 \mathrm{~h}$ of urine collection. $\mathrm{AKI}+=\mathrm{AKI}$ stage 2 or 3 at $12 \mathrm{~h}$ of urine collection. ICU = intensive care unit. $\mathrm{UACE} 2=$ urinary angiotensin-converting enzyme type $2 . \mathrm{uCr}=\mathrm{urinary}$ creatinine. * Panel A compares UACE2 activity in ICU patients with AKI stage 0 or 1 at $12 \mathrm{~h}$ of urine collection, ICU patients with AKI stage 2 or 3 at 12 h of urine collection and healthy volunteers, with $P$ values shown for betweengroup differences. UACE2 activity was significantly higher in ICU patients without AKI stage 2 or 3 at 12 h of urine collection, compared with the other two groups. Panel B shows UACE2 levels based on AKI stages (no AKI, $n=55$; AKI stage 1, $n=18$; AKI stage 2, $n=30$; AKI stage $3, n=2$ ), with the $P$ value representing significance of the association between UACE2 activity with AKI stage. In both figures, the $y$-axis was log-transformed to improve readability, and individual data and boxplots (representing median values, interquartile ranges, minimum and maximum values) are shown.

activity compared with those without severe AKI. Moreover, we found that their UACE2 levels were comparable to those of healthy volunteers, and were half the levels seen in those who did not develop severe AKI. Finally, UACE2 showed potential as a biomarker for predicting early severe $\mathrm{AKI}$ in ICU patients, demonstrating high sensitivity yet low specificity for the primary outcome.

\section{Relationship to previous studies}

This study is the first and only report on UACE2 measurements in critically ill patients at risk of AKI. It adds a novel line of evidence regarding the role of the RAS in critical illness, and renal failure more specifically. Our results suggest that the non-classical RAS may play a role in ICU patients at risk of severe AKI. It is plausible that the non-classical RAS counterbalances the systemic and local effects of the classical RAS on inflammation and intrarenal haemodynamics, by increasing the generation

levels correctly reclassified $28 \%$ of patients to a higher risk category of the primary outcome (positive NRI) (Table 3).

After determining an optimal cut-off point, we found that a UACE2 activity below $12.8 \mathrm{pmol} / \mathrm{min} / \mathrm{mL}$ per $\mathrm{mmol} / \mathrm{L}$ of $\mathrm{uCr}$ had a sensitivity of $81 \%(95 \% \mathrm{Cl}, 64-93 \%)$ and a specificity of $51 \%(95 \% \mathrm{Cl}, 39-63 \%)$ to predict the primary outcome (see Online Appendix, table S4, for crosstabulation and other parameters of diagnostic accuracy).

\section{Discussion \\ Main findings}

In a prospective cohort of ICU patients, we measured UACE2 activity levels and assessed their association with the development of severe AKI. We found that patients who developed severe AKI had significantly lower UACE2 of angiotensin (1-7) and (1-9). ${ }^{37}$ Indeed, our findings are congruent with experimental evidence showing that inhibited generation of pivotal peptides of the non-classical cascade increases kidney injury in ACE2 knock-out mice after experimental ischemia-reperfusion. ${ }^{38}$

To put our results into further physiological perspective, increases in urinary excretion of renin and angiotensinogen were observed in patients with severe AKI, while classical RAS overexpression (angiotensin ॥ and angiotensinogen) was demonstrated in renal biopsy specimens of patients with proven acute tubular necrosis. ${ }^{39-41}$ On the other hand, we can hypothesise that non-classical pathway activation inducing an increased angiotensin ${ }^{1-7}$ response may protect from AKI, as suggested by preclinical research. ${ }^{16,20}$ This is indeed suggested by our data, as patients experiencing a 


\section{ORIGINAL ARTICLES}

Figure 3. Association between UACE2 levels and study outcomes*

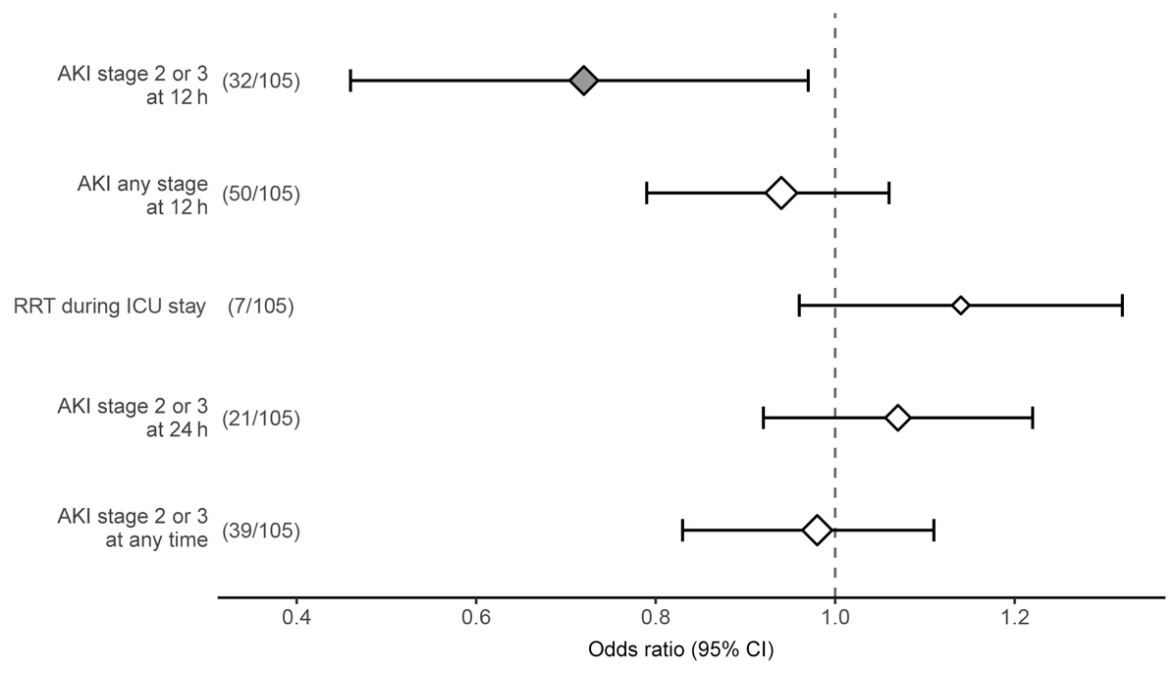

$A K I=$ acute kidney injury. ICU = intensive care unit. RRT = renal replacement therapy. $\mathrm{UACE} 2=$ urinary angiotensinconverting enzyme type $2 . \mathrm{uCr}$ = urinary creatinine. * The figure shows the unadjusted odds ratios (with $95 \% \mathrm{Cls}$ ) for the primary outcome (AKI stage 2 or 3 at $12 \mathrm{~h}$ ) and the secondary renal outcomes, for an increase in UACE2 level of $10 \mathrm{pmol} / \mathrm{min} / \mathrm{mL}$ per $\mathrm{mmol} / \mathrm{L}$ of $\mathrm{uCr}$. The size of each diamond is proportional to the number of events (indicated in parentheses). and angiotensin-(1-9)

Angiotensin-(1-7), the pivotal peptide of the nonclassical cascade, decreases renal blood flow, increases natriuresis, and inhibits proinflammatory and fibrosis pathways in experimental models of AKI. ${ }^{10,18-20}$ Hence, we hypothesise that in patients with upregulated ACE2 activity, the increase in angiotensin II bioavailability (through intravenous administration) would explain the benefits on RRT weaning observed in the post-hoc analysis of the Angiotensin II for the Treatment of High-Output Shock (ATHOS-3) trial. ${ }^{43}$ However, the trial only reported markers of the classical pathway activation.

Finally, neither ACE critical insult who did not develop AKI showed increased excretion of UACE2, and there was a trend in lower UACE2 levels being associated with the most severe cases. Conversely, those who did develop AKI showed levels comparable to those of healthy volunteers, suggesting their inability to activate this potentially protective endocrine response in the face of a renal insult. Also, increased angiotensin II levels, by increasing tubular sodium reabsorption and reducing renal blood flow, may have decreased diuresis, which would explain the higher incidence of oliguria-defined AKI in our cohort.

These findings highlight the potential importance of classical/non-classical RAS balance in the pathogenesis of AKI. However, our study did not analyse other markers of classical or non-classical pathways, such as angiotensin II or angiotensin-(1-7) levels in blood or urine, and this limits our ability to draw solid conclusions.

From a clinical point of view, a recent post-hoc analysis of a large randomised controlled trial of angiotensin II administration in patients with vasodilatory shock and severe AKI showed that drug-induced classical RAS upregulation accelerated RRT weaning rates. ${ }^{42}$ While this may appear to challenge our hypothesis, it is biologically plausible that systemic angiotensin II infusion may have increased perfusion pressure and, after metabolism by ACE2, generated much higher levels of angiotensin-(1-7) inhibitor use nor angiotensin receptor blocker use affected UACE2 levels in our cohort, which is in line with experimental data showing their inability to inhibit ACE2. ${ }^{44}$

\section{Implications of study findings}

Our results imply that non-classical RAS upregulation may play a role in the development of severe AKI in ICU patients. Our findings generate new pathophysiological hypotheses regarding the development of $\mathrm{AKI}$, that are congruent with the preclinical evidence of the effects of non-classical RAS upregulation on renal blood flow, natriuresis, inflammatory processes and profibrotic processes. ${ }^{18,20,45,46}$ Based on our preliminary results, the ability of $U A C E 2$ to predict AKI should be explored further. Moreover, these preliminary findings imply that additional efforts should be made to understand how classical and non-classical RAS interact during AKI pathogenesis. Finally, based on the experimental and clinical experience in acute lung injury, our data imply that the effect of recombinant human ACE2 on AKI should be evaluated at the preclinical stage first. ${ }^{47,48}$ This is especially relevant in the context of the SARS-CoV-2 pandemic, because SARS-CoV-2 causes ACE2-mediated viral disease. Indeed, critically ill patients infected with SARS-CoV-2 have high incidence of severe AKI, which we hypothesise could, to some level, be related to deficiency of ACE2 activity induced by the virus. ${ }^{49}$ 


\section{ORIGINAL ARTICLES}

Figure 4. Log-rank analysis of risk of AKI based on UACE2 levels*

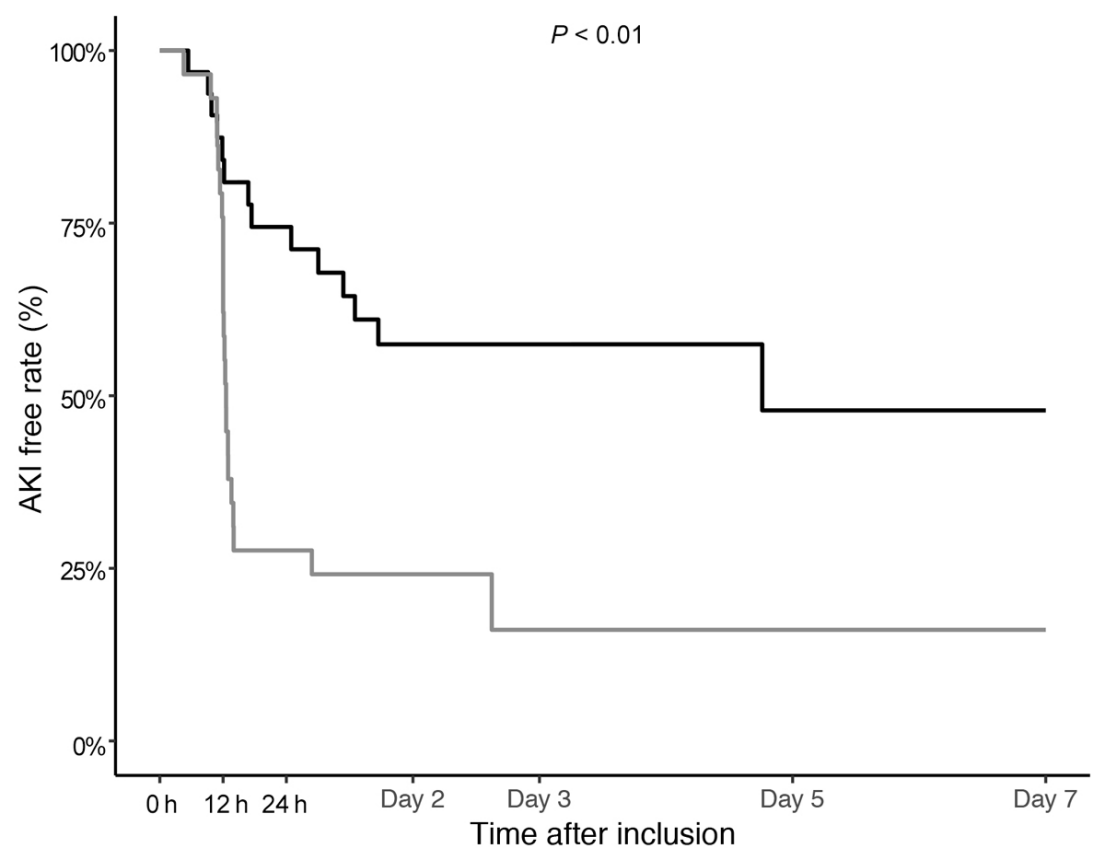

$A K I=$ acute kidney injury. ICU = intensive care unit. UACE2 = urinary angiotensin-converting enzyme type 2. $\mathrm{uCr}=$ urinary creatinine. ${ }^{*}$ The Kaplan-Meier graph shows the AKI-free (non-stage 2 or 3) rate over 7 days in all patients who had not developed AKI (any stage) before urine collection $(n=76)$. We divided those patients into two groups, based on the median value of UACE2 levels in the cohort (8.7 $\mathrm{pmol} / \mathrm{min} / \mathrm{mL}$ per $\mathrm{mmol} / \mathrm{L}$ of $\mathrm{UCr}$ ). The P value represents the difference in AKI incidence, using the logrank method, until censor date (ICU discharge, Day 7 after urine collection or death). Patients who had UACE2 levels below the median UACE2 value had a significantly higher risk of developing AKI stage 2 or 3 during ICU stay (grey line) compared with those who had UACE2 levels above the median (black line).

biomarkers in the context of acute fluctuation of glomerular filtration rates and urine flow, although it may have led to overestimation of UACE2 sensitivity. ${ }^{28,29}$ Finally, we used adequate and validated statistical methods, including NRIs and multivariate models, to assess the predictive performance of UACE2.

However, some limitations should be acknowledged. First, this was a single-centre study, which limits the external validity of the findings. However, our ICU has all the characteristics of a tertiary teaching centre, including a broad spectrum of both medical and postoperative patients. Also, we observed an incidence of severe AKI similar to that reported in other large studies. ${ }^{3}$

Second, this was an observational study, limiting any inference on causality. Moreover, the absence of association with the predefined secondary outcomes mandates cautious interpretation of our findings. However, our results on the association of UACE2 activity with severe and early $A K I$ are in line with what would be expected based on previous work and our current

\section{Strengths and limitations}

Our study has several strengths. It is the first to report UACE2 activity levels in a general population of critically ill patients, and to compare their findings with those for healthy volunteers. We enrolled a large number of critically ill patients for whom there was a high incidence of severe $\mathrm{AKI}$ - a reflection of our study population's severity of illness. Also, although a majority of identified AKI episodes were classified using isolated oliguria in our study, low urine output is known to be associated with worse clinical outcomes in ICU patients, even if transient or in the absence of a creatinine increase. ${ }^{50-52}$

Another strength was the fact that UACE2 activity was measured by an investigator who was blinded to the clinical context and all investigators were unaware of the UACE2 results at the time of AKI adjudication. Also, UACE2 activity was corrected for urine creatinine concentration, similar to the methods used to quantify urinary angiotensinogen. ${ }^{39}$ This correcting method is recommended when assessing urine understanding of the RAS. Furthermore, we showed a significant association of UACE2 with the incidence of severe AKI in our time-dependent analysis, suggesting the importance of timing when evaluating a potential biomarker in relation to a clinical process. We also acknowledge that UACE2 as a biomarker showed discrimination of limited performance in our population, although its sensitivity was high. We believe that this is at least partly due to our study's casemix - that is, of a general ICU population.

Third, it is plausible that UACE2 levels may have been affected by increased glomerular filtration rates and/ or plasma protein leakage, and may not have reflected intrarenal production. However, ACE2 filtration through the glomerular membrane seems unlikely, as its molecular weight is about $120 \mathrm{kDa}$ - twice that of albumin. ${ }^{53}$ Also, this has been highlighted as a potential source of bias in patients with chronic kidney disease $\mathrm{H}^{46}$ and was accounted for by correcting for urinary creatinine levels. Apart from urine concentration and glomerular filtration, UACE2 could be affected by urine output in this population of patients 


\section{ORIGINAL ARTICLES}

with both oliguric and non-oliguric AKI, owing to effects of circadian rhythm in our cohort of healthy volunteers. However, we confirmed our findings when assessing UACE2 activity in patients without oliguria at time of inclusion.

Fourth, we found that our study, although significant for its primary outcome, had potentially low statistical power. The risk of such a situation is to overestimate the effect size of our results. Testing the hypothesis that the effect size was half of what we observed, there was a $6 \%$ risk that the sign (direction) of the effect was wrong (a type S error) and that its magnitude could be four times lower (type M error). ${ }^{54}$

Finally, using NRI values to assess the performance of a biomarker may depend strongly on the quality of the reference model. In our study, the reference model was extrinsically valid, and its performance confirmed, using recommended methods.

\section{Conclusions}

In this exploratory study of patients at high risk of AKI, higher UACE2 activity was associated with a decreased risk of developing severe AKI and showed significant, yet limited, performance for predicting the incidence of severe AKI. Our hypothesis-generating study warrants further investigations to explore the complex roles of the classical and non-classical RAS in the pathogenesis of AKI.

Acknowledgements: We thank Leah Peck, Helen Young and all Austin Health ICU staff for the help and support provided for this project. The ACE2 assay costs were supported by a National Health and Medical Research Council Program Grant (APP1055214) awarded to Louise Burrell.

\section{Competing interests}

None declared.

\section{Author details}

Laurent Bitker ${ }^{1,2}$

Sheila K Patel ${ }^{3}$

Intissar Bittar ${ }^{4}$

Glenn M Eastwood ${ }^{1}$

Rinaldo Bellomo ${ }^{1,5}$

Louise M Burrell ${ }^{3}$

1 Department of Intensive Care, Austin Health, Melbourne, VIC, Australia.

2 Université de Lyon, Lyon, France.

3 Department of Medicine, Austin Health, Melbourne, VIC, Australia.

4 Department of Pathology, Austin Health, Melbourne, VIC, Australia

5 Centre for Integrated Critical Care, University of Melbourne, Melbourne, VIC, Australia.

Correspondence: laurent.bitker@chu-lyon.fr

\section{References}

1 Ostermann M, Liu K. Pathophysiology of AKI. Best Pract Res Clin Anaesthesio/ 2017; 31: 305-14.

2 Nisula S, Kaukonen KM, Vaara ST, et al. Incidence, risk factors and 90-day mortality of patients with acute kidney injury in Finnish intensive care units: the FINNAKI study. Intensive Care Med 2013; 39: 420-8.

3 Hoste EA, Bagshaw SM, Bellomo R, et al. Epidemiology of acute kidney injury in critically ill patients: the multinational AKI-EPI study. Intensive Care Med 2015; 41: 1411-23.

4 Chertow GM, Burdick E, Honour M, et al. Acute kidney injury, mortality, length of stay, and costs in hospitalized patients. J Am Soc Nephrol 2005; 16: 3365-70.

5 Bellomo R, Kellum JA, Ronco C. Acute kidney injury. Lancet 2012; 380: 756-66.

6 Calzavacca P, Evans RG, Bailey M, et al. Cortical and medullary tissue perfusion and oxygenation in experimental septic acute kidney injury. Crit Care Med 2015; 43: e431-9.

7 Calzavacca P, May CN, Bellomo R. Glomerular haemodynamics, the renal sympathetic nervous system and sepsis-induced acute kidney injury. Nephrol Dial Transplant 2014; 29: 2178-84.

8 Sparks MA, Crowley SD, Gurley SB, et al. Classical renin-angiotensin system in kidney physiology. Compr Physiol 2014; 4: 1201-28.

9 Chappell MC. Nonclassical renin-angiotensin system and renal function. Compr Physiol 2012; 2: 2733-52.

10 Bitker L, Burrell LM. Classic and nonclassic renin-angiotensin systems in the critically ill. Crit Care Clin 2019; 35: 213-27.

11 Donoghue $\mathrm{M}$, Hsieh $\mathrm{F}$, Baronas $\mathrm{E}$, et al. A novel angiotensinconverting enzyme-related carboxypeptidase (ACE2) converts angiotensin I to angiotensin 1-9. Circ Res 2000; 87: E1-9.

12 Chappell MC. Emerging evidence for a functional angiotensinconverting enzyme 2-angiotensin-(1-7)-MAS receptor axis: more than regulation of blood pressure? Hypertension 2007; 50: 596-9.

13 Burrell LM, Risvanis J, Kubota E, et al. Myocardial infarction increases ACE2 expression in rat and humans. Eur Heart J 2005; 26: 369-75; discussion 22-4.

14 Mizuiri S, Hemmi H, Arita M, et al. Expression of ACE and ACE2 in individuals with diabetic kidney disease and healthy controls. Am J Kidney Dis 2008; 51: 613-23.

15 Hoffmann M, Kleine-Weber H, Schroeder S, et al. SARS-CoV-2 cell entry depends on ACE2 and TMPRSS2 and is blocked by a clinically proven protease inhibitor. Cell 2020; 181: 271-80.e8.

16 Chen $\sqcup, X u Y L$, Song $B$, et al. Angiotensin-converting enzyme 2 ameliorates renal fibrosis by blocking the activation of mTOR/ERK signaling in apolipoprotein E-deficient mice. Peptides 2016; 79: 49-57.

17 Gwathmey TM, Westwood BM, Pirro NT, et al. Nuclear angiotensin-(1-7) receptor is functionally coupled to the formation of nitric oxide. Am J Physiol Renal Physiol 2010; 299: F983-90.

18 Heller J, Kramer HJ, Maly J, et al. Effect of intrarenal infusion of angiotensin-(1-7) in the dog. Kidney Blood Press Res 2000; 23: 89-94.

19 Li P, Chappell MC, Ferrario CM, Brosnihan KB. Angiotensin-(1-7) augments bradykinin-induced vasodilation by competing with ACE and releasing nitric oxide. Hypertension 1997; 29: 394-400.

20 Pinheiro SV, Ferreira AJ, Kitten GT, et al. Genetic deletion of the angiotensin-(1-7) receptor Mas leads to glomerular hyperfiltration 


\section{ORIGINAL ARTICLES}

and microalbuminuria. Kidney Int 2009; 75: 1184-93.

21 Lew RA, Warner FJ, Hanchapola I, Smith Al. Characterization of angiotensin converting enzyme-2 (ACE2) in human urine. Int $J$ Pept Res Ther 2006; 12: 283-9.

22 von Elm E, Altman DG, Egger $M$, et al. The Strengthening the Reporting of Observational Studies in Epidemiology (STROBE) statement: guidelines for reporting observational studies. J Clin Epidemiol 2008; 61: 344-9.

23 Bossuyt PM, Reitsma JB, Bruns DE, et al. STARD 2015: an updated list of essential items for reporting diagnostic accuracy studies. BMJ 2015; 351: h5527.

24 Vincent JL, Moreno R, Takala J, et al. The SOFA (sepsis-related organ failure assessment) score to describe organ dysfunction/failure. On behalf of the Working Group on Sepsis-Related Problems of the European Society of Intensive Care Medicine. Intensive Care Med 1996; 22: 707-10.

25 Kashani K, Al-Khafaji A, Ardiles T, et al. Discovery and validation of cell cycle arrest biomarkers in human acute kidney injury. Crit Care 2013; 17: R25.

26 KDIGO Clinical Practice Guideline for Acute Kidney Injury. Kidney Int Supp/ 2012; 2: 1-138.

27 Toh L, Bitker L, Eastwood GM, Bellomo R. The incidence, characteristics, outcomes and associations of small short-term point-of-care creatinine increases in critically ill patients. J Crit Care 2019; 52: 227-32.

28 Tonomura Y, Uehara T, Yamamoto E, et al. Decrease in urinary creatinine in acute kidney injury influences diagnostic value of urinary biomarker-to-creatinine ratio in rats. Toxicology 2011; 290: 241-8.

29 Waikar SS, Sabbisetti VS, Bonventre JV. Normalization of urinary biomarkers to creatinine during changes in glomerular filtration rate. Kidney Int 2010; 78: 486-94.

30 Calzavacca P, Tee A, Licari E, et al. Point-of-care measurement of serum creatinine in the intensive care unit. Ren Fail 2012; 34: 13-8.

31 Levey AS, Bosch JP, Lewis JB, et al. A more accurate method to estimate glomerular filtration rate from serum creatinine: a new prediction equation. Modification of Diet in Renal Disease Study Group. Ann Intern Med 1999; 130: 461-70.

32 Knaus WA, Wagner DP, Draper EA, et al. The APACHE III prognostic system. Risk prediction of hospital mortality for critically ill hospitalized adults. Chest 1991; 100: 1619-36.

33 Therneau TM. A package for survival analysis in S. Version 2.38, 2015.

34 Robin X, Turck N, Hainard A, et al. pROC: an open-source package for $\mathrm{R}$ and $\mathrm{S}+$ to analyze and compare ROC curves. $B M C$ Bioinformatics 2011; 12: 77.

35 R Development Core Team. R: a language and environment for statistical computing. Vienna, Austria: R Foundation for Statistical Computing, 2008.

36 Pencina MJ, D'Agostino RB, Sr, D’Agostino RB, Jr, Vasan RS. Evaluating the added predictive ability of a new marker: from area under the ROC curve to reclassification and beyond. Stat Med 2008; 27: 157-72; discussion 207-12.

37 Zhang J, Noble NA, Border WA, Huang Y. Infusion of angiotensin-(1-7) reduces glomerulosclerosis through counteracting angiotensin II in experimental glomerulonephritis. Am J Physiol Renal Physiol 2010; 298: F579-88.

38 Fang F, Liu GC, Zhou $X$, et al. Loss of ACE2 exacerbates murine renal ischemia-reperfusion injury. PLoS One 2013; 8: e71433.

39 Alge JL, Karakala N, Neely BA, et al. Association of elevated urinary concentration of renin-angiotensin system components and severe AKI. Clin J Am Soc Nephrol 2013; 8: 2043-52.

40 Alge JL, Karakala N, Neely BA, et al. Urinary angiotensinogen and risk of severe AKI. Clin J Am Soc Nephrol 2013; 8: 184-93.

41 Cao W, Jin L, Zhou Z, et al. Overexpression of intrarenal reninangiotensin system in human acute tubular necrosis. Kidney Blood Press Res 2016; 41: 746-56.

42 Tumlin JA, Murugan R, Deane AM, et al. Outcomes in patients with vasodilatory shock and renal replacement therapy treated with intravenous angiotensin II. Crit Care Med 2018: 46: 949-57.

43 Bellomo R, Wunderink RG, Szerlip $\mathrm{H}$, et al. Angiotensin I and angiotensin II concentrations and their ratio in catecholamineresistant vasodilatory shock. Crit Care 2020; 24: 43.

44 Towler P, Staker B, Prasad SG, et al. ACE2 x-ray structures reveal a large hinge-bending motion important for inhibitor binding and catalysis. J Biol Chem 2004; 279: 17996-8007.

45 Jin HY, Chen LJ, Zhang ZZ, et al. Deletion of angiotensinconverting enzyme 2 exacerbates renal inflammation and injury in apolipoprotein E-deficient mice through modulation of the nephrin and TNF-alpha-TNFRSF1A signaling. J Transl Med 2015; 13: 255.

46 Mizuiri S, Aoki T, Hemmi H, et al. Urinary angiotensin-converting enzyme 2 in patients with CKD. Nephrology (Carlton) 2011; 16: 567-72.

47 Imai Y, Kuba K, Rao S, et al. Angiotensin-converting enzyme 2 protects from severe acute lung failure. Nature 2005; 436: 112-6.

48 Khan A, Benthin C, Zeno B, et al. A pilot clinical trial of recombinant human angiotensin-converting enzyme 2 in acute respiratory distress syndrome. Crit Care 2017; 21: 234.

49 Richardson S, Hirsch JS, Narasimhan M, et al. Presenting characteristics, comorbidities, and outcomes among 5700 patients hospitalized with COVID-19 in the New York City area. JAMA 2020; 323: 2052-9.

50 Macedo E, Bouchard J, Soroko SH, et al. Fluid accumulation, recognition and staging of acute kidney injury in critically-ill patients. Crit Care 2010; 14: R82.

51 Macedo E, Malhotra R, Bouchard J, et al. Oliguria is an early predictor of higher mortality in critically ill patients. Kidney Int 2011; 80: 760-7.

52 Vaara ST, Parviainen I, Pettila V, et al. Association of oliguria with the development of acute kidney injury in the critically ill. Kidney Int 2016; 89: 200-8.

53 Tipnis SR, Hooper NM, Hyde R, et al. A human homolog of angiotensin-converting enzyme. Cloning and functional expression as a captopril-insensitive carboxypeptidase. J Bio/ Chem 2000; 275: 33238-43.

54 Gelman A, Carlin J. Beyond power calculations: assessing type S (sign) and type M (magnitude) errors. Perspect Psychol Sci 2014; 9: 641-51. 